\title{
Utilización del programa de visualización molecular RasMol como estrategia didáctica para la integración del contenido curricular 'proteínas'
}

\author{
M.A. Riera, M. Caldez, E.M. Giorgio, L.B. Milde, P.D. Zapata
}

Introducción. Los programas de visualización permiten profundizar el conocimiento teórico sobre la estructura proteica. Este trabajo tiene por finalidad familiarizar al alumno con el manejo del programa de visualización molecular RasMol v. 2.7.2 para el análisis de proteínas, ya que integra conocimientos previos y persigue otros objetivos generales de la formación universitaria. Materiales y métodos. El trabajo se contextualizó considerando el eje integrador de las materias. Además, se articuló con los contenidos conceptuales y procedimentales de la temática 'Niveles estructurales de las proteínas'. En primer lugar, se seleccionaron actividades que permitieran evaluar aspectos vinculados con la formación general pretendida para este nivel de la enseñanza formal, como expresión escrita y oral, interpretación de textos cientíicos en idioma inglés y manejo de base de datos bibliográficos. Resultados y conclusiones. El 70\% de los alumnos pudo realizar las actividades de manera satisfactoria. La mayoría de los grupos enfatizó tanto las cuestiones teóricas como las netamente inherentes al manejo del programa. El 30\% tuvo problemas de expresión oral y escrita. Se notaron algunas dificultades en la interpretación de los modelos teóricos planteados y en la interpretación del idioma inglés, pero también hubo desconocimiento previo de la temática y falta de iniciativa para la búsqueda de material complementario. Se propone la inclusión de algunas revisiones en idioma nativo, sin perjuicio de los textos en inglés, y ejemplos más sencillos de manera introductoria para los programas de visualización, con una paulatina incorporación de ejemplos más complejos que requieran lectura complementaria.

Palabras clave. Biología celular y molecular. Programas de visualización. Proteínas. Recursos didácticos.

\section{Utilization of the molecular visualization program RasMol as a didactic strategy for the integration of the curricular content 'proteins'}

Introduction. Visualizations programs allow to analyze deeply theoretical knowledge of protein structure. The aim of this work was to familiarize the students with the molecular visualization program RasMol v. 2.7.2 for protein analysis, integrating previous concepts and pursuing other general objectives of university formation. Materials and methods. The work was contextualized considering the integrative axis of the subjects, articulating it with conceptual and procedural contents of the topic 'Protein Structure Levels.' Activities were selected in order to evaluate different aspects of the general formation pretended at this level of teaching, like oral and written expression, interpretation of scientific papers in English, and bibliographic database search. Results and conclusions. $80 \%$ of the students were able to do the proposed activities with acceptable quality. Most of the students equally emphasized both the theoretical and practical procedures. $30 \%$ of the students have shown problems with oral or written expression. We noticed some difficulties in the interpretation of the proposed theoretical models and interpretation of the scientific papers in English. Additionally students did not show previous knowledge of the topics and did not look up for complementary bibliography. We propose to include some reviews in native language and simpler examples to serve as an introduction to the visualization program, with gradual incorporation of more complex models that require complementary bibliography reading.

Key words. Didactic resources. Molecular and cellular biology. Proteins. Visualization programs.

\author{
Cátedra de Biología \\ Celular y Molecular. \\ Módulo de Bioquímica \\ y Farmacia. Facultad de \\ Ciencias Exactas Químicas \\ y Naturales. Universidad \\ Nacional de Misiones. \\ Posadas, Misiones, \\ Argentina.

\section{Correspondencia} \\ Dr. Pedro Darío Zapata. \\ Cátedra de Biología \\ Celular y Molecular. \\ Módulo de Bioquímica \\ y Farmacia. Facultad de \\ Ciencias Exactas Químicas \\ y Naturales. Universidad \\ Nacional de Misiones. \\ Avda. Mariano Moreno, 1375. \\ 3300 Posadas, Misiones, \\ Argentina. \\ E-mail \\ pdr_dario@yahoo.com
}

Agradecimientos A los docentes M. Guastavino, A. Paroli, J.C. Ortiz Márquez, M.I. Fonseca y M. Ruiz Díaz, por su colaboración al realizar las actividades descritas. 


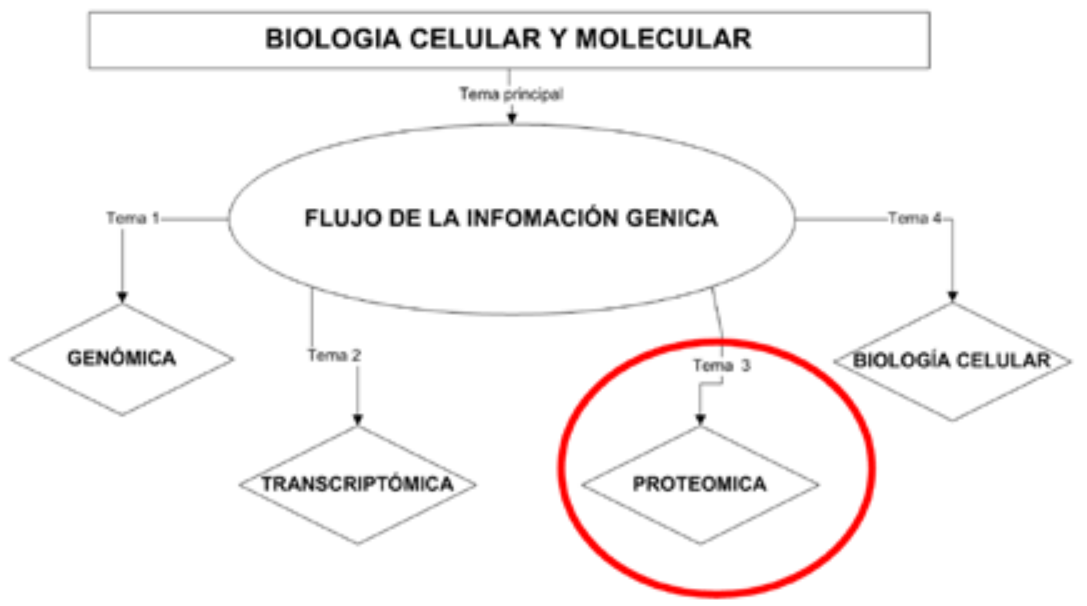

Figura 1. Mapa conceptual del tema 'Flujo de la información génica' en el que se enmarcan los contenidos conceptuales y procedimentales del trabajo práctico impartido (círculo) dentro del área 'Biología celular y molecular' abordada por ambas asignaturas.

\section{Introducción}

Las proteínas son biomoléculas de gran importancia en el funcionamiento celular y determinantes de la amplia diversidad de procesos y variantes que presentan las células. El análisis comprensivo de su estructura tridimensional amplía la posibilidad de integrar los saberes previos a los nuevos conceptos impartidos en cada tema [1].

Los programas de visualización son una herramienta sumamente útil en este sentido y se encuentran disponibles de manera libre en la web, siendo además muy utilizados en diversos trabajos científicos para el análisis de moléculas. Además, su uso se relaciona con el manejo de información científica publicada en las diferentes bases de datos disponibles [2]. En otras universidades, la utilización del programa RasMol permite al alumnado una asimilación de los conocimientos relacionados con la estructura de las biomoléculas [3].

El presente trabajo persigue como competencia específica familiarizar al alumno con el manejo del programa de visualización molecular RasMol v. 2.7.2 ( ${ }^{\odot}$ Herbert Bernstein, 1998-2000) para el análisis de proteínas. Con esto se pretende integrar de manera práctica los conocimientos previamente adquiridos en forma teórica, addemás de perseguir otras competencias transversales de la formación universitaria, como el manejo de la información científica y bibliográfi- ca específica de una temática, propiciar el manejo de información accesible mediante Internet, fomentar el uso de recursos didácticos para la presentación gráfica y visual de la información requerida, y elaborar informes escritos sobre una temática específica.

La materia 'Biología molecular y genética' es cuatrimestral y se da en el tercer año de la carrera de Bioquímica, mientras que 'Biología celular' es una materia cuatrimestral que se imparte en el segundo año de la carrera de licenciatura en Genética; ambas abordan el estudio de la biología celular y molecular. La ubicación curricular de las materias permite utilizar conceptos previos del alumno sobre la estructura química de las biomoléculas y su metabolismo, ya que es uno de los objetivos de estas materias profundizar e integrar la estructura y función de las biomoléculas en los diferentes procesos celulares, y establecer así un punto de partida previo para el análisis del flujo de la información génica. Ambas materias son dictadas por el mismo equipo docente. Se detectó en años anteriores la falta de profundización en la estructura proteica, así como la falta de relación entre los aspectos teóricos y la aplicación práctica [4]. Es necesario transmitir correctamente, de forma acotada y efectiva, la utilización de una nueva herramienta [5], de manera que el alumno logre esclarecer fallos conceptuales o preconceptos teóricos con esta aplicación práctica [6]. 


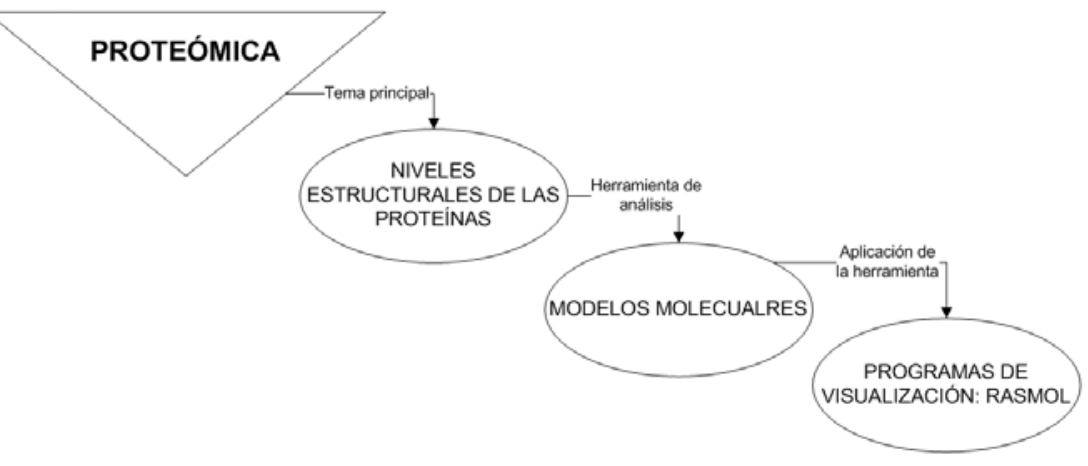

Figura 2. Contextualización curricular de la temática del trabajo práctico propuesto. Se muestra el desglose de los aspectos teóricos y prácticos incluidos en el tema principal que aborda la propuesta.

\section{Materiales y métodos}

\section{Ubicación curricular de los contenidos}

Considerando el eje integrador de la materia, el tema 'proteínas' fue seleccionado para su abordaje como trabajo práctico (Fig. 1).

\section{Propuesta de enseñanza-aprendizaje}

Acorde con el tema seleccionado (proteínas), se planteó un trabajo práctico semipresencial titulado 'Utilización de programas de modelizacion molecular como estrategia didáctica para el estudio de las proteínas', que se contextualizó curricularmente dentro de los contenidos procedimentales de la temática 'Niveles estructurales de las proteínas' (Fig. 2).

\section{Programa-guía de actividades propuesta}

Las actividades se seleccionaron para permitir evaluar competencias específicas y transversales, vinculadas con la formación general pretendida para este nivel de la enseñanza formal, es decir, expresión escrita y oral, interpretación de textos científicos en idioma inglés y manejo de base de datos bibliográficos.

Los temas seleccionados fueron: canales de potasio, transportadores de glucosa y bombas de calcio. Se entregó una guía temática con un tutorial para la presentación del informe. Este material se digitalizó y vinculó al aula virtual de la asignatura (URL: http://www.aulavirtualexactas.dyndns.org/biocelmol), y se propuso la modalidad de trabajo grupal hasta cinco integrantes.

Las guías temáticas elaboradas contenían una breve introducción teórica al tema, objetivos del trabajo práctico, actividades propuestas, autoevaluación, bibliografía sugerida y modelo de informe. Las actividades consistieron en la ubicación de los dominios estructurales y funcionales de la proteína, mostrando en cada caso la posición de algunos aminoácidos fundamentales de estas estructuras. Las imágenes debían incorporarse al informe, junto con las respuestas de las preguntas de autoevaluación.

Para el desarrollo del trabajo práctico se ajustó la siguiente planificación:

\section{Etapa 1}

Clase presencial de iniciación a la temática y familiarización con el programa $(2 \mathrm{~h})$. Se realizó durante la segunda semana de clases:

- Presentación de los objetivos del trabajo práctico.

- Explicación del funcionamiento del aula virtual.

- Explicación del funcionamiento del programa RasMol usando como ejemplo la molécula de hemoglobina.

- Explicación del acceso a base de datos para la obtención de información. 


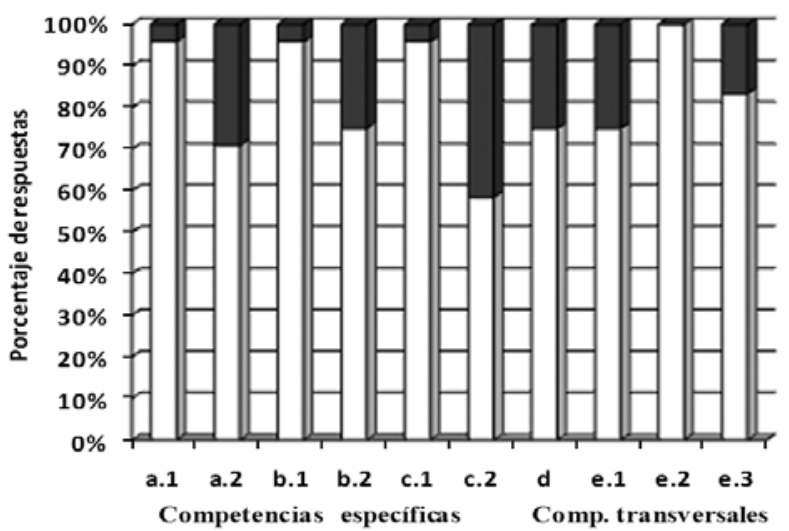

Figura 3. Evaluación del desempeño de los estudiantes para las actividades propuestas. Competencias específicas: a.1) Manejo del programa de visualización, instancia oral; a.2) Manejo del programa de visualización, informe escrito; b.1) Enfoque práctico de la presentación, instancia oral; b.2) Enfoque práctico de la presentación, informe escrito; c.1) Enfoque teórico de la presentación, instancia oral; c.2) Enfoque teórico de la presentación, informe escrito. Competencia transversales: d) Expresión escrita: calidad general de la presentación; e.1) Expresión oral: claridad de la presentación oral; e.2) Recursos informáticos de presentación e.3) Distribución del tiempo de exposición. Las barras en blanco indican 'satisfactorio', y las barras en negro, 'no satisfactorio'.

\section{Etapa 2}

Profundización del manejo conceptual y procedimental a través de la realización de tareas no presenciales por los alumnos. Estas tareas debían entregarse en el plazo de un mes a partir a la clase presencial.

\section{Etapa 3}

Análisis y discusión de los resultados del trabajo práctico. Al cumplirse el plazo de entrega de resultados, los alumnos realizaron una presentación oral, con una duración de 10 minutos por grupo, que coincidió con la entrega del informe escrito.

\section{Evaluación del desempeño de los estudiantes para las actividades propuestas}

Se realizó a través de las presentaciones orales y los informes escritos. Para la evaluación se consideraron aspectos relacionados con las competencias específicas y con las competencias transversales:

\section{Competencias específicas}

(60\% de la evaluación)

- Manejo del programa de visualización: instancia oral e informe escrito.
- Enfoque práctico de la presentación: instancia oral e informe escrito.

- Enfoque teórico de la presentación: instancia oral e informe escrito.

\section{Competencias transversales}

(40\% de la evaluación)

- Expresión escrita: calidad general de la presentación

- Expresión oral: claridad de la presentación oral, recursos informáticos de presentación, y distribución del tiempo de exposición (presenta - muestra - concluye).

\section{Análisis estadístico de los datos}

Durante las presentaciones orales se registraron los aspectos a evaluar para cada grupo de alumnos. Los informes se corrigieron siguiendo las pautas evaluativas. Las respuestas se agruparon de acuerdo con la categoría de las competencias evaluadas (específicas o transversales) y se categorizaron como satisfactorias y no satisfactorias. Se dispusieron en gráficas los porcentajes alcanzados por el total de alumnos para cada competencia. 


\section{Resultados}

En la versión escrita del informe presentado por los alumnos se previeron dos correcciones. Se mandó rehacer diez trabajos por no ajustarse a las consignas solicitadas, mientras que tres grupos no presentaron los informes por escrito a pesar de serles reclamados. Estos grupos fueron eliminados del estudio, quedando un total de 24 grupos. Los resultados finales que arrojó la evaluación del desempeño de los estudiantes se muestran en la figura 3, distribuidos por competencias.

\section{Discusión}

Los ítems que evalúan las competencias generales apuntan a considerar la capacidad de interpretación del tema abordado, durante el trabajo práctico por parte del alumnado, mediante la utilización del programa de visualización como herramienta para llevar a cabo las actividades previstas.

Mediante las actividades previstas, el alumno obtuvo diferentes pantallas que le permitían visualizar y ejemplificar los aspectos teóricos de la estructura proteica. En este sentido, la evaluación del informe escrito da cuenta de los logros alcanzados al intentar analizar los aspectos teóricos de la estructura proteica con el uso del programa, mientras que la evaluación de presentación oral se centró en valorar la capacidad de asociación entre teoría y práctica. Alrededor del $70 \%$ pudo realizar las actividades de manera satisfactoria (regular + bueno). Sin embargo, en las cuestiones teóricas, solo un $60 \%$ fue satisfactorio. Esta observación podría relacionarse con algunas dificultades en la interpretación de los modelos teóricos planteados o en la interpretación del idioma inglés, dado que el material de lectura se aportó en dicho idioma, conforme a las recomendaciones realizadas por la Comisión Nacional de Evaluación y Acreditación Universitaria, en la resolución de evaluación prevista para la carrera de Bioquímica de la Universidad Nacional de Misiones [7]. Por último, si se suma el desconocimiento previo de la temática y la falta de iniciativa para la búsqueda de material complementario en su idioma, ello nos lleva a proponer la inclusión de algunas revisiones en idioma nativo, sin perjuicio de los textos en inglés. Por otro lado, la falta de conocimiento profundo de la temática podría solucionarse en estos casos con ejemplos más sencillos de manera introductoria, de modo que el análisis de información para asistir la interpretación de las gráficas de los programas de visualización se vea facilitada y se incluyan así, gradualmente, ejemplos más complejos que requieran lectura complementaria.

La materia está ubicada en los primeros años de la carrera y se deben evaluar algunos aspectos transversales, como la expresión oral y escrita. La presentación oral y los informes permiten valorar estos aspectos. Alrededor de un 30\% de los alumnos tuvo problemas de expresión oral o escrita, por lo que sugerimos continuar trabajando estas competencias en otras materias de los currículos.

De acuerdo con lo expresado por Arcá [8], aunque las propuestas concretas, desarrolladas y experimentadas a veces son aceptadas y se plantean en nuevas clases, su significado no pasa de ser episódico y puntual. Por ello, es importante la articulación entre materias para permitir una apropiación más profunda de la herramienta planteada (programas de visualización) y generar otras instancias de evaluación que aporten una mirada general de las competencias transversales que se evalúan en este trabajo práctico.

La aplicación de esta metodología de trabajo no intenta resolver los problemas cognitivos disciplinarios y didácticos del tema, pero propone posibles caminos de cambio a fin de influir más profundamente en la formación y educación científica, estimulando una actitud de apertura consciente mediante la utilización de herramientas informáticas para el análisis de las proteínas.

\section{Bibliografía}

1. Lodish H, Berk A, Zipursky S, Matsudaira P, Baltimore D, Darnell J. Estructura y función de las proteínas. In Lodish H, Berk A, Zipursky S, Matsudaira P, Baltimore D, Darnell J, eds. Biología celular y molecular. Madrid: Médica Panamericana; 2002. p. 50-99.

2. Claros-Díaz MG, Ávila-Sáez C, Gallardo-Alba F, CánovasRamos FM. Metodología relacionada con la informática. In Claros-Díaz MG, Ávila-Sáez C, Gallardo-Alba F, CánovasRamos FM, eds. Bioquímica aplicada: manual para el diseño experimental y el análisis de datos en bioquímica y biología molecular. Oviedo: Septem Ediciones; 2001. p. 231-62. 
3. Herráez-Sánchez A, Tejedor-Gilmartín MC. Biomodel. In: Ayudas al aprendizaje de bioquímica, biotecnología y biología molecular. BioROM 2006. URL: http://www2.uah. es/biomodel. [12.05.2009].

4. Cariaga-Martínez AE, Zapata PD. Aplicación de un trabajo práctico autoguiado para la formación en el uso de herramientas bioinformáticas de alumnos de pregrado en Bioquímica Clínica. Educ Med 2006; 9: 9-13.

5. McNamara D. Subject knowledge and its application: problems and possibilities for teacher educators. J Educ Teach 1991; 17: 113-28.
6. Wandersee JH, Mintzes JJ, Novak JD. Research on alternative conceptions in science. In Gabel DL, ed. Handbook of research on science teaching and learning. New York: Macmillan; 1994. p. 117-210.

7. Resolución 334/07 (Exp n..$^{\circ}$ 804-077/06). Comisión Nacional de Evaluación y Acreditación Universitaria (Ministerio de Educación). URL: http://www.fceqyn.unam.edu.ar/ acreditacion-bioquimica/archivos/res334-07E80407706. pdf. [12.05.2009].

8. Arcá M. Una propuesta para la formación en aula de ciencias. Revista de Educación en Biología-ADBiA 2005; 8: 8-17. 